\title{
RETROPERITONEOSCOPY FOR TREATMENT OF RENAL AND URETERAL STONES
}

\author{
RODRIGO S. SOARES, PEDRO ROMANELLI, MARCOS A. SANDOVAL, MARCELO M. \\ SALIM, JOSE E. TAVORA, DAVID L. ABELHA JR \\ Section of Urology, Hospital da Previdência dos Servidores do Estado de Minas Gerais, Belo Horizonte, \\ Minas Gerais, Brazil
}

\begin{abstract}
Objective: To assess the efficacy of retroperitoneoscopy for treating stones in the renal pelvis and proximal ureter.

Materials and Methods: In the period from August 2003 to August 2004, 35 retroperitoneoscopies for treatment of urinary stones were performed on 34 patients. Fifteen patients $(42 \%)$ had stones in the renal pelvis, and in 2 cases, there were associated stones in the upper caliceal group. Twenty patients (58\%) had ureteral stones, all of them located above the iliac vessel. Twenty-five patients (71\%) had previously undergone at least one session of extracorporeal lithotripsy and 8 patients $(26 \%)$ also underwent ureteroscopy to attempt to remove the stone. Eight patients underwent retroperitoneoscopy as a primary procedure. Stone size ranged from 0.5 to $6 \mathrm{~cm}$ with a mean of $2.1 \mathrm{~cm}$.

Results: Retroperitoneoscopy was performed by lumbar approach with initial access conducted by open technique and creation of space by digital dissection. We used a 10-mm Hasson trocar for the optics, and 2 or 3 additional working ports placed under visualization. Following identification, the urinary tract was opened with a laparoscopic scalpel and the stone was removed intact. The urinary tract was closed with absorbable 4-0 suture and a Penrose drain was left in the retroperitoneum. In 17 patients (49\%), a double-J stent was maintained postoperatively. Surgical time ranged from 60 to 260 minutes with a mean of 140 minutes. The mean hospital stay was 3 days (1-10 days). The mean length of retroperitoneal urinary drainage was 3 days (1-10 days). There were minor complications in $6(17.6 \%)$ patients and 1 case of conversion due to technical difficulty. Thirty-three patients $(94 \%)$ became stone free.

Conclusion: Retroperitoneoscopy is an effective, low-morbidity alternative for treatment of urinary stones.
\end{abstract}

Key words: kidney calculi; ureteral calculi; surgery; laparoscopy; retroperitoneal space

Int Braz J Urol. 2005; 31: 111-6

\section{INTRODUCTION}

Recent advances in extracorporeal lithotripsy and endoscopic techniques have made open surgery infrequent for treatment of urinary stones. However, patients with large impacted stones, especially those located in the proximal ureter, pose a challenge for treatment, often requiring multiple interventions. In such cases, open surgery remains a good, cost-effective alternative for resolution. The recent development of laparoscopic surgery has broadened the therapeutic options for several pathologies. The laparoscopic ureterolithotomy was initially described by Wickhan (1) in 1979, and more widely divulged since the early 1990 s by Gaur (2), using the retroperitoneoscopic access. The present study reports 
the experience with retroperitoneoscopic ureterolithotomy and pyelolithotomy.

\section{PATIENTS AND METHODS}

Over the period from August 2003 to August 2004, 34 patients ranging from 14 to 65 years of age (mean 32 years) underwent 35 retroperitoneoscopic interventions for removal of urinary stones. Nineteen patients were male and 15 were female. Twenty-four presented microscopic hematuria and/or lumbar pain, 4 patients had recurrent urinary infection and 5 patients were asymptomatic. One patient presented obstructive acute renal failure with anuria and anasarca due to bilateral stones.

In all patients, the diagnosis was confirmed with imaging exams (plain abdominal x-ray, ultrasonography and excretory urography or abdominal computerized tomography). Twenty-four patients presented moderate or more pronounced hydronephrosis due to the presence of stones. Two patients presented ureteropelvic junction stenosis with associated stones. Two patients had complete ureteropelvic duplicity with ureteral stones in the upper collecting system. Twenty-nine patients had radiopaque stones and 5 had radiolucent ones. Stones were located on the right side in 17 patients, on the left side in 16 patients, and 1 patient had bilateral lithiasis.

Among the 34 patients, 31 had a single stone and 3 had 2 or more stones or stone fragments. Twenty patients had ureteral stones, with 14 located in the proximal ureter and 6 located in the middle ureter. Fifteen patients presented renal stones, with 13 cases of single pelvic stones and 2 cases of pelvic stones associated with stones in the upper caliceal group. Twentyfive patients had previously undergone at least 1 session of extracorporeal lithotripsy, and 7 patients also underwent ureteroscopy in an attempt to remove the stone. One patient underwent ureteroscopy only, which was unsuccessful. In 8 patients, retroperitoneoscopy was indicated as the primary treatment. Eight patients presented previously inserted double-J stents. One patient had a nephrostomy. Stone size ranged from 0.5 $\mathrm{cm}$ to $6 \mathrm{~cm}$ in diameter (mean $2.1 \mathrm{~cm}$ ).

The main indication for laparoscopy was as an alternative to open and/or percutaneous surgery following failure of extracorporeal lithotripsy and/or ureterolithotripsy. The condition for indicating retroperitoneoscopy for ureteral stones was a location above the iliac vessels, while the presence of an extra-renal or dilated pelvis provided the condition for pelvic stones. The procedure was also indicated in 2 cases with stones located in the upper calyx due to anatomical aspects that favored extraction through the renal pelvis. Two patients with UPJ stenosis underwent retroperitoneoscopic pyeloplasty during the same surgical procedure. No patient presented previous lumbotomy. The data for study analysis were collected retrospectively. All patients signed an informed consent term.

\section{Surgical Technique}

The procedure was a lumbar retroperitoneoscopic under general anesthesia in all patients. In 5 cases, a double-J stent was positioned in retrograde way during the immediate preoperative period. Patients were positioned in the classic lumbotomy position with hyperextension. Access to the retroperitoneal space was obtained with the open technique through a $1.5-\mathrm{mm}$ subcostal incision under the extremity of the 12th rib and muscle divulsion up to the aponeurosis of the transverse muscle, which was then opened, and the fascia transversalis, identifying the prerenal fat. The creation of a working space in the retroperitoneum was performed by digital dissection, displacing the peritoneum medially without using a balloon. A Hasson trocar was inserted in this space and fixed to the musculature with a purse-string suture in order to avoid air leakage and development of subcutaneous emphysema, and $\mathrm{CO}_{2}$ insufflation was performed until reaching 12-mm $\mathrm{Hg}$ tension. We used 0degree optics and, when needed, the working space was completed using the optics under visualization. Two additional trocars were placed under visualization; a 5-mm trocar on the hemiclavicular line just above the iliac spine and caudally to the optics port, and a 5- or 10-mm trocar posterior to the optics at the posterior axillary line, forming a triangle. Eventually, when retraction was required, another 5-mm trocar was placed at an anterior position at the hemiclavicular line just below the costal margin (Figure-1). 


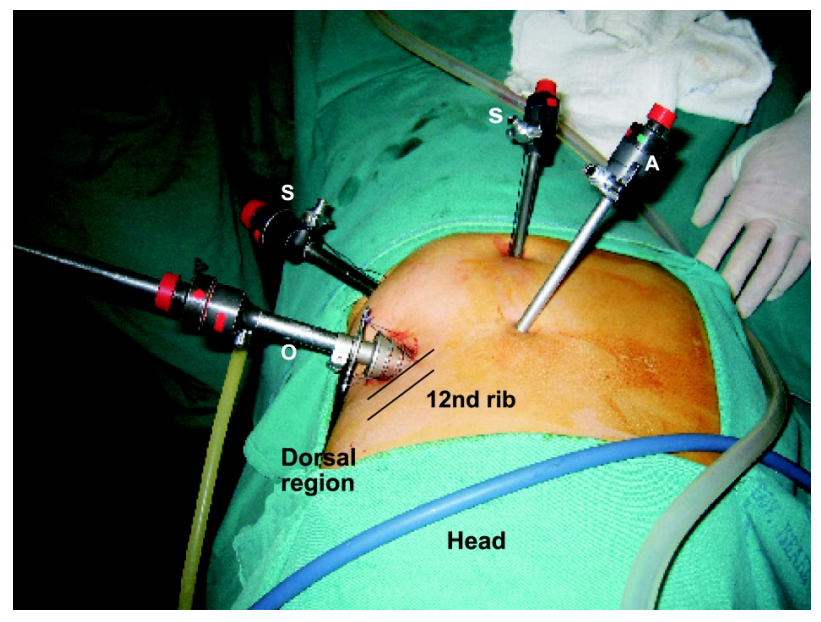

Figure 1 - Upper view of a patient in right lateral decubitus showing the location of the ports $(S=$ surgeon, $A=$ assistant, $O=$ optics).

The ureter was initially identified in its middle portion within the retroperitoneal fat and dissected up to the level of the UPJ. Identifying the stone was also relatively easy in cases with upstream dilation of the urinary tract. Two cases required the use of radioscopy. The accurate location of the intra-ureteral stone was achieved by palpation with laparoscopic forceps.

In the majority of cases, the urinary tract was opened with a longitudinal incision using a laparoscopic scalpel. At the beginning of our experience, due to the unavailability of tools, the opening was performed using laparoscopic scissors or diathermy and a metallic curve needle held by laparoscopic forceps. Stone extraction was performed using laparoscopic forceps only. In both cases with stones in the upper caliceal group, the stone was extracted with a rigid nephroscope inserted through an Amplatz sheath placed at the incision site for the optic port after removing the Hasson trocar. The nephroscope was guided to the renal pelvis by a guide wire that had been previously positioned through pyelotomy and the stone was removed using transnephroscopic forceps under saline solution irrigation. In cases where it was feasible, the stone was immediately removed from the cavity using the inner area of one of the trocars without contacting the wall. Large stones were temporarily kept in the retroperi- toneal space and, at the end of the surgery, the stone was introduced into a bag made out of a surgical glove and then removed.

The double-J stent was positioned during the surgical procedure in 9 cases, during the immediate peroperative period by retrograde approach in 5 cases and during the peroperative period by antegrade approach in 4, using a guide wire through one of the 5$\mathrm{mm}$ ports. The indication for inserting the stent in the peroperative period was if there was complete obstruction of the urinary tract due to a long-term impacted stone or the technical difficulty of achieving a satisfactory closure of the renal pelvis or ureter at the beginning of our experience.

Suture of the urinary tract was performed in all cases, except, due to technical difficulties, in one case of pyelolithotomy. In the first 10 cases, closure was performed with single stitches using non-absorbable 4-0 polyglycolic acid sutures; the other cases used 4-0 chromic catgut. Eventually the continuous suture was performed in wider pyelotomies. A Penrose drain was placed in the retroperitoneum and exteriorized through one of the port incisions, and was subsequently removed when the drainage was lower than $30 \mathrm{~mL} / 24$ hours. The ureteral catheter was removed on average 3 weeks after the procedure and a radiographic control was performed around the 30 th postoperative day.

\section{RESULTS}

Stone removal using the retroperitoneoscopic approach was successfully accomplished in 33 of the 35 interventions. In one case, conversion to open surgery was required due to technical difficulties. In another case of ureteral stone with a previously inserted double-J stent, it was impossible to locate the stone even with radioscopic aid. This patient subsequently underwent a new ureterosocopy, achieving stone removal.

Surgical time ranged from $60 \mathrm{~min}$ to $260 \mathrm{~min}$ (mean 140). Peroperative bleeding was negligible, except in 2 patients that presented bleeding of approximately $500 \mathrm{~mL}$ due to damage to the left gonadal vein and 1 parietal artery, respectively. In both cases, bleeding was controlled without requiring con- 
version to open surgery. One patient presented a voluminous retroperitoneal hematoma in the postoperative period, which was treated conservatively. No case required blood transfusion, the hospital stay ranged from 1 to 10 days (mean 3 ) and the follow-up ranged from 2 to 12 months (mean 7). All patients became stone-free and no case of urinary tract stenosis was observed during the follow-up.

The length of urinary drainage through the Penrose drain ranged from 1 to 10 days (mean 3 days), being more prolonged in cases where the urinary tract was not opened with laparoscopic scalpel, in cases without double-J stent and in 1 case with double-J stent and in 1 case where the pyelotomy was not sutured. In 2 cases with abundant postoperative drainage, the postoperative insertion of a double-J stent allowed the closure of the fistula in 1 day. Postoperative complications occurred in $6(17.6 \%)$ patients. Two patients presented an abscess at the port site where the Penrose drain was exteriorized. One patient who had previous nephrostomy developed urinary sepsis during the postoperative period following inadvertent removal of the nephrostomy and improved after inserting a retrograde ureteral catheter. One patient evidenced retroperitoneal hematoma and was treated conservatively. One patient presented pain and paresthesia in the ipsilateral lumbar region due to a thermal lesion of the intercostal nerve during efforts to control the bleeding of the intercostal artery, and was clinically managed. Another patient presented subcutaneous emphysema due to subcutaneous insufflation of $\mathrm{CO}_{2}$ with no clinical repercussions.

\section{COMMENTS}

Despite the development of extracorporeal lithotripsy and advancements in endoscopic techniques for treatment of urinary stones, some patients still undergo open surgery (3). The main surgical indications in our setting result from failure and/or unavailability of minimally invasive techniques. The recent advancement of laparoscopy in the urologic field has permitted a new alternative for treatment of stones (4).

Traditionally the access for stone removal in open surgery is achieved by retroperitoneal approach.
Some authors advocate laparoscopic surgery for managing stones using the intraperitoneal approach (4-7) since it has the advantage of providing a larger working space. The retroperitoneoscopic approach spread widely following the use of a balloon for creating a working space in a study developed by Gaur $(2,8)$. This approach provides direct access to the urinary tract and avoids manipulation and contact of urine with the intraperitoneal organs. The main disadvantage of retroperitoneoscopic access is the smaller working space, which renders reconstructive procedures such as suturing of the urinary tract more difficult (8). In our experience, the use of a balloon was not required for creating the retroperitoneal space. Using only digital dissection and complementing the dissection with the optic itself when required, we obtained a space that allowed us to fully perform the procedure with satisfactory laparoscopic sutures. Positioning the surgeon's working ports caudally to the optics makes the procedure easier.

The lumbar retroperitoneoscopic approach allows a fast and direct access to the urinary tract from the renal pelvis to the ureter at the level of the iliac vessels. In favorable conditions, it is possible to obtain access to stones located in caliceal groups, especially the upper groups, through the renal pelvis. In 2 cases it was possible to extract upper caliceal stones using a rigid nephroscope that was inserted through an opening in the renal pelvis. Those cases had dilated calices and wide infundibulum, which made the procedure easier. The combination of laparoscopic and percutaneous nephrolithotripsy techniques has already been described, especially for treating stones in ectopic kidneys (9). However, the present study shows the possibility of successfully combining these 2 techniques to extract caliceal stones in selected cases. The possibility of accessing the stones using a transpelvic approach reduces the risk of bleeding compared to the transparenchymal approach. This is an advantage of retroperitoneoscopy over percutaneous nephrolithotomy. The selection of patients with extra-renal and/or a dilated renal pelvis was fundamental for obtaining good results with the retroperitoneoscopy. Another advantage is the possibility of removing the stone intact with no fragmentation and, thus, lower the risk of residual fragments. 
In all cases in this series the stone was extracted intact, even in cases where the stone measured more than $5 \mathrm{~cm}$. At the moment, few studies have been published comparing the classic open approach with retroperitoneoscopy (10). Other studies are still required in order to assess each method's advantages; however, the advancement in laparoscopic techniques and instruments enables us to widen the applications of retroperitoneoscopy $(11,12)$.

Flexible urethroscopes or even flexible cystoscopes using lasers (13-15) can also be used as a resource for application by retroperitoneoscopic approach, similarly to what was performed in our study with the rigid nephroscope, thus increasing the device's scope and the chances of success in selected cases.

Whether to use the ureteral catheter or not is a controversial issue in urinary stone surgery (16). The presence of a double-J stent makes identification of the ureter in the retroperitoneum easier, however it makes the identification of the stone difficult since it reduces the dilation of the urinary tract, and they can be easily mistaken because of the stent's rigidity. In one case from this series, in a patient with a radiolucent ureteral stone, the presence of a previously inserted double-J stent prevented the stone from being located.

In cases with anatomic changes to the urinary tract, the laparoscopy represents a safe and effective alternative to endoscopic procedures, which are often laborious and risky in such cases. In 2 cases with pyeloureteral duplicity, the laparoscopic access enabled easy localization and extraction of the stone after ureteroscopy efforts had been frustrated. Similarly, in 2 cases with ureteropelvic junction stenosis associated with stones in the dilated pelvis, laparoscopy enabled easy stone extraction, and reconstruction with pelvic reduction, reproducing the conventional open surgery (17).

Laparoscopy is a method that reproduces the steps of open surgery and can be indicated as an alternative in cases of therapeutic failure using less invasive methods $(18,19)$. However, in cases where the risk of failure using such method is high, such as anatomic anomalies and voluminous and impacted ureteral stones, laparoscopy can be indicated as a primary procedure (8). In cases of pelvic stones, the retroperitoneoscopic pyelolithotomy is an alternative that allows the removal of the intact stone with lower risk of residual fragments and without requiring transparenchymal access, thus reducing the risk of bleeding.

\section{REFERENCES}

1. Wickham JEA: The Surgical Treatment of Urinary Lithiasis. In Wickham JEA (ed.), Urinary Calculus Disease. New York, Churchill Livingstone. 1979; pp. 145-98.

2. Gaur DD: Retroperitoneal laparoscopic ureterolithotomy. World J Urol. 1993; 11: 175-7.

3. Assimos DG, Boyce WH, Harrison LH, McCullough DL, Kroovand RL, Sweat KR: The role of open stone surgery since extracorporeal shock wave lithotripsy. J Urol. 1989; 142: 263-7.

4. Raboy A, Ferzli GS, Ioffreda R, Albert PS: Laparoscopic ureterolithotomy. Urology. 1992; 39: 223-5.

5. Harewood LM, Webb DR, Pope AJ: Laparoscopic ureterolithotomy: the results of an initial series, and an evaluation of its role in the management of ureteric calculi. Br J Urol. 1994; 74: 170-6.

6. Micali S, Moore RG, Averch TD, Adams JB, Kavoussi LR: The role of laparoscopy in the treatment of renal and ureteral calculi. J Urol. 1997; 157: 463-6.

7. Keeley FX, Gialas I, Pillai M, Chrisofos M, Tolley DA: Laparoscopic ureterolithotomy: the Edinburgh experience. BJU Int. 1999; 84: 765-9.

8. Gaur DD, Trivedi S, Prabhudesai MR, Madhusudhana HR, Gopichand M: Laparoscopic ureterolithotomy: technical considerations and long-term follow-up. BJU Int. 2002; 289: 339-43.

9. Holman E, Toth C: Laparoscopically assisted percutaneous transperitoneal nephrolithotomy in pelvic dystopic kidneys: experience in 15 successful cases. J Laparoendosc Adv Sur Tech A. 1998; 8: 431-5.

10. Goel A, Hemal AK: Evaluation of role of retroperitoneoscopic pyelolithotomy and its comparison with percutaneous nephrolithotripsy. Int Urol Nephrol. 2003; 35: 73-6.

11. Gaur DD, Trivedi S, Prabhudesai MR, Gopichand M: Retroperitoneal laparoscopic pyelolithotomy for staghorn stones. J Laparoendosc Surg Tech A. 2002; 12: 299-303.

12. Kaouk JH, Gill IS, Desai MM, Banks KL, Raja SS, Skarel M, et al.: Laparoscopic anathrophic nephroli- 
thotomy: feasibility study in a chronic porcine model. J Urol. 2003; 169: 691-6.

13. Chow GK, Patterson DE, Blute ML, Segura JW: Ureteroscopy: effect of technology and technique on clinical practice. J Urol. 2003; 170: 99-102.

14. Kerbl K, Rehman J, Landman J, Lee D, Sundaram C, Clayman RV: Current management of urolithiasis: progress or regress? J Endourol. 2002; 16: 281-8.

15. Landman J, Lee DI, Lee C, Monga M: Evaluation of overall cost and currently available small flexible ureteroscopes. Urology. 2003; 62: 218-22.

16. Chew RH, Knudson BE, Deustedt JD: The use of stents in contemporary urology. Curr Opin Urol. 2004; 14: 111-5.
17. Ramakumar S, Lancini V, Chan DY, Parsons JK, Kavoussi LR, Jarrett TW: Laparoscopic pyeloplasty with concomitant pyelolithotomy. J Urol. 2002; 167: 1378-80.

18. Skrepetis K, Doumas K, Siafakas I, Lykourinas M: Laparoscopic versus open ureterolithotomy. A comparative study. Eur Urol. 2001; 40: 32-6.

19. Goel A, Hemal AK: Upper and mid-ureteric stones: a prospective unrandomized comparison of retroperitoneoscopic and open ureterolithotomy. BJU Int. 2002; 89: 635-6.

\section{Correspondence address:}

Dr. Rodrigo S. Quintela Soares

Rua Aluminio 50 / 31

Belo Horizonte, MG, 30220-090, Brazil

Phone: + $55313222-2666$

E-mail: quintelarod@yahoo.com

Received: October 6, 2004

Accepted after revision: January 13, 2005 